\title{
Resonant ionization of shallow donors in electric field
}

Ivan Gueorguiev Ivanov and Erik Janzén

\section{Linköping University Post Print}

N.B.: When citing this work, cite the original article.

Original Publication:

Ivan Gueorguiev Ivanov and Erik Janzén, Resonant ionization of shallow donors in electric field, 2014, Physica Scripta, (89), 8, 085802.

http://dx.doi.org/10.1088/0031-8949/89/8/085802

Copyright: IOP Publishing: Hybrid Open Access

http://www.iop.org/

Postprint available at: Linköping University Electronic Press

http://urn.kb.se/resolve?urn=urn:nbn:se:liu:diva-111496 


\title{
Resonant ionization of shallow donors in electric field
}

\author{
I G Ivanov and E Janzén \\ Department of Physics, Chemistry and Biology, Linköping University, Linköping, 58183 \\ Sweden \\ E-mail: ivani@ifm.liu.se
}

\begin{abstract}
We report experimental observation of resonant ionization of the phosphorus donor in silicon in homogeneous electric field, expressed in sudden rise of the conductivity of the sample at low temperature when the electric field approaches the critical value of $\sim 3.2 \mathrm{MV} / \mathrm{m}$. The effect is discussed in terms of field-induced interaction of the states on the ground of a simplified model based on the effective-mass theory. The results from our model are qualitatively similar to previously published advanced model based on first principles, which predicts ionization thresholds at approximate fields 2.45 and $3.25 \mathrm{MV} / \mathrm{m}$, the latter being in very good agreement with our experiment. The possibility for observation of more than one resonance is also discussed.
\end{abstract}

PACS: 71.55.-i, 71.70.-d, 71.70.Ej

\section{Introduction}

Many works starting already at the dawn of quantum mechanism have been studying the dc Stark effect in the hydrogen atom or its analogue, a shallow donor in a semiconductor. Recent renewed interest in the field is due to the proposed by Kane [1] quantum computer, which requires that the hyperfine interaction of the donor electron with the nucleus is governed by an external electric field. In contemporary work almost exclusively devoted to the phosphorus (P) donor in silicon several approaches are used to calculate the dependence of the energy spectrum of the donor electron on the electric field. Among these it is worth mentioning the complex scaling [2], a variational method [3], and expansion of the wave functions in a Gaussian basis set in combination with first principle calculations [4]. The effect of the valley-orbit (VO) interaction, which couples one-valley wave functions and leads to splitting of the ground state (mainly), is neglected in [2] but accounted for in the other two works, albeit in different ways. While Friesen [3] introduces phenomenological parameters to fit the ground-state splitting to the experimental values, Debernardi et al. [4] employ the core potentials of Si and P obtained from first principles. Representing the zero-cell potential as a difference between the potentials of P and Si they obtain at zero electric field a ground-state manifold very similar to the experimentally observed one. Most importantly, however, this work [4] discusses ionization of the $1 s\left(\mathrm{~A}_{1}\right)$ level (the ground state) of the donor at an electric field $\sim 2.45$ $\mathrm{MV} / \mathrm{m}$ due to anti-crossing of the ground state with a $p$-like state. In the present paper we report the first, to the best of our knowledge, experimental observation of such ionization, and give further account on this effect, which may be properly addressed as resonant ionization in electric field. Qualitative discussion of the experimental result is conducted in terms of field-induced interaction of the donor states. The latter is discussed in the frame of a simplified theoretical model based on the effective-mass theory (EMT). Our model does not account for the valley-orbit splitting and the central cell potential, and does not provide sufficient accuracy for quantitative comparison with experiment, but it bears some qualitative similarity with the advanced calculation of Ref. [4], that is why it is used as a background for discussion of the experiment in terms of interaction of the states. Quantitative comparison of the experimental data with the predictions of the more accurate theory of Ref. [4] is carried out.

\section{Theoretical background}

The ionization threshold around $2.45 \mathrm{MV} / \mathrm{m}$ discussed in detail in Ref. [4] is a consequence of two factors. The first factor encounters the obvious circumstance that higher excited states are subject to ionization at 
lower values of the external electric field due to their larger orbits and less localization at the binding donor nucleus. At higher electric fields the wave function describing given excited state is well delocalized, i.e., its integrated amplitude inside the well formed by the Coulomb potential of the donor is much smaller than the corresponding integrated amplitude outside the well, and in this case the energy of the state depends almost linearly on the applied electric field, similar to the energy of a free electron [5]. This is demonstrated in Ref. [4], in particular for the states forming the $2 s$ and $2 p$ manifolds at zero field. Already at electric field of few hundred $\mathrm{kV} / \mathrm{m}$, their energies reach above the ionization threshold defined in Ref. [4] as the minimum barrier of the potential well surrounding the impurity, formed by the Coulomb potential of the donor and the superimposed electric field (cf. figure 1 of Ref. [4]). However, at higher electric field (e.g., $~ 1.6 \mathrm{MV} / \mathrm{m}$ for the $2 \mathrm{p}_{0}$ states) their energies are again under the ionization threshold; this peculiar behaviour is discussed in [4] in detail. The second factor concerns the interaction of the states. At electric field such that the energy of an excited state (e.g., $\left.2 \mathrm{p}_{0}\right)$ tends to intersect the energy of a lower state (in particular, the ground state), a strong mixing of the two states occurs provided that they have the same symmetry, i.e., transform as the same irreducible representation of the symmetry group of the Hamiltonian. The mixing (or interaction) of the states stipulates the anti-crossing behaviour in the energy versus field dependence of the two interacting states (cf. [4]), but also leads to "exchange" of their character. That is, the bound state becomes unbound and turns to linear energy dependence on the electric field, while the unbound state becomes bound. The relatively narrow region of electric fields where anticrossing occurs corresponds to the critical field of Ref. [4]. The exchange in the character of the anticrossing states is only briefly mentioned in [4], however, it provides us with a reason to anticipate that the ionization bears resonant character, i.e., the ionization probability decays both below and above the critical field, because of the existence of binding state both below and above the critical field. This behaviour is discussed further below.

We shall illustrate the above reasoning in the framework of a simplified model for a shallow donor in silicon (e.g., phosphorus) within the EMT in the one-valley approximation (i.e., neglecting the valley-orbit interaction). Although the model will be shown to be rather inaccurate from quantitative point of view and overlooks effects associated with the central-cell and valley-orbit interactions, it provides sufficient background for illustration of the field-induced mixing (interaction) of the states. We only consider the behaviour of the ground state of the donor electron in presence of homogeneous electric field along the (001) direction. The one-valley Schrödinger equation for the envelope function $\Phi$ in spherical coordinates $(\rho, \theta, \varphi)$ reads

$$
\left(-\Delta-\frac{2}{\rho} f(\theta)-\gamma \sigma \rho \cos \theta\right) \Phi(\rho, \theta, \varphi)=\mathrm{E} \Phi(\rho, \theta, \varphi) .
$$

Here $\mathrm{E}$ is the corresponding energy (in units of the effective Rydberg $R_{\text {eff }}$ defined below), $\sigma=\left(m_{T} / m_{L}\right)^{1 / 2}$, where $m_{T}=0.191$ and $m_{L}=0.916$ are the transversal and longitudinal effective masses in units of the free-electron mass, and $\Delta$ is the Laplace operator. The function $f(\theta)=1 /\left(1-\alpha \cos ^{2} \theta\right)^{1 / 2}$ describes the anisotropy, and $\alpha=1-m_{T} / m_{L}$. The units of energy (E) and length ( $\rho$ ) are defined by the effective Rydberg $R_{\text {eff }}$ and the effective Bohr radius $a_{\text {eff }}$, respectively:

$$
R_{e f f}=\frac{m_{T}}{\varepsilon^{2}} \frac{e^{4}}{2 \hbar^{2}}, \text { and } a_{e f f}=\frac{\varepsilon}{m_{T}} \frac{\hbar^{2}}{e^{4}} .
$$

Here $e$ is the electron charge and $\varepsilon$ - the static dielectric constant. The "field factor" $\gamma$ is defined by $\gamma=e F a_{\text {eff }} / R_{\text {eff }}$, where $F$ is the amplitude of the applied homogeneous electric field.

In our model the wave function $\Phi$ is expanded in a series of the normalized hydrogen wave functions $\varphi_{n l m}(\rho, \theta, \varphi)=R_{n l}(\rho) Y_{l}^{m}(\theta, \varphi)$, which provide exact solutions of Eq.(1) with $f(\theta) \equiv 1$ and $\gamma=0$. Here $R_{n l}(\rho)$ is the radial part of the wave functions with quantum numbers $n, l$ and $m$, whereas $Y_{l}^{m}(\theta, \varphi)$ denote the spherical harmonics. This choice is by no means the best choice from point of view 
of convergence of the series, which reflects on the accuracy of the calculated eigenstates. However, it provides a convenient way for analyzing the energy-level interactions. We note that since the Hamiltonian in the presence of electric field is unbound, methods using boundary conditions (e.g., as in Ref.[6]) are inappropriate. Variational methods similar to the method of Ref.[3] are not suitable either, since they rely on a trial function (in fact, superposition of only $1 s$ and $2 p_{0}$ in [3]) and the interaction of the states is overlooked.

The group of symmetry of the Hamiltonian in Eq.(1) in the absence of electric field $(F=0)$ is $\mathrm{D}_{\text {och }}$ and reduces to $\mathrm{C}_{\infty \mathrm{v}}$ for $F \neq 0$ along one of the (100) axes of the crystal. The cylindrical symmetry of the Hamiltonian implies that the magnetic quantum number $m$ remains a good quantum number also in the latter case, hence all $s$-like (fully-symmetric) states among which is the ground state are represented by

$$
\Phi^{S}(\rho, \theta, \varphi)=\sum_{n=1}^{20} \sum_{l=0}^{n-1} \alpha_{n l}^{S} \varphi_{n l}(\rho, \theta, \varphi),
$$

but with different sets of the coefficients $\left\{\alpha_{n l}^{S}\right\}$ for the different states enumerated by the superscript $S$ (in a common notation, $1 s, 2 p_{0}, 2 s$, etc.). Here we omit the quantum number $m=0$. Note that in the presence of electric field the $1 s$ and the $2 p_{0}$ states are both fully-symmetric due to the symmetry reduction from $\mathrm{D}_{\infty \mathrm{ch}}$ to $\mathrm{C}_{\infty \mathrm{v}}$ and, therefore, are allowed to mix (and similarly for higher $p$-like states with $m=0$ ). Wishing to investigate the ground-state behaviour, we restrict our calculation to the fully-symmetric states. It is worth noting, however, that the calculation of Ref. [4] includes also states of other symmetries (one of them is represented by the straight line in the insert of their figure 2) which, however, do not interact with the ground state.

Using the expansion (3) including all functions $\varphi_{n l m}$ with main quantum number $n$ up to 20 (210 functions), we solve Eq. (1) in a standard manner and obtain for the ground 1s state a binding energy of $29.5 \mathrm{meV}$ at zero field $(F=0)$, to be compared with the accurate value of $31.2 \mathrm{meV}[7,8]$, i.e., a relative error of $\sim 6 \%$.

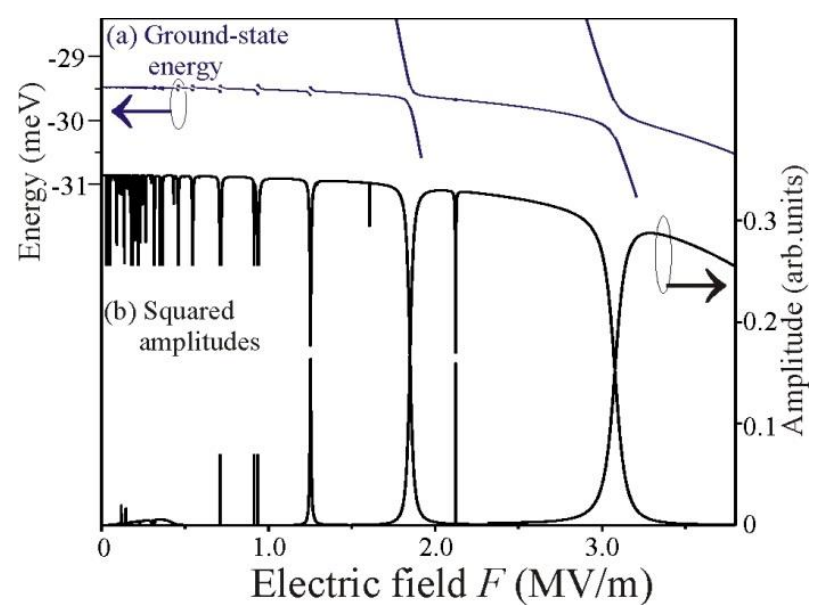

Figure 1. Results of the calculation within our model. (a) Variation of the ground-state energy with the electric field $F$. The energies of the two anti-crossing states near 1.8 and $3.1 \mathrm{MV} / \mathrm{m}$ discussed in text are plotted extended around the anti-crossing point in order to show the linear parts. (b) Variation of the squared amplitudes at the nucleus for the ground state (upper curve) and the nearest excited state (lower curve).

\section{Theore tical and experimental results and discussion}

\subsection{Interpretation of the theoretical results}

The results of the calculation are illustrated in figure 1. It should be noted that the present model suffers several deficiencies which will be outlined shortly, therefore within our model we shall only discuss the behaviour of the wave functions of the two well-localized states near the anti-crossing points at $\sim 1.8$ and 
3.1 MV/m [cf. figure 1(a)], which will be argued to correspond to the anti-crossings at 2.45 and 3.25 $\mathrm{MV} / \mathrm{m}$ observed in [4], despite of the disagreement in the critical-field values. Let us regard first the dependence of the energy of the ground state on the electric field [figure 1(a)]. This dependence exhibits multiple discontinuities, many of which occur at low electric fields and are not observed in the energy versus field dependence of Ref. [4]. We attribute most of the discontinuities occurring at low fields to artefacts of the use of very extended but limited basis, which leads to the appearance of many artificial states in the calculation, for which the series expansion, Eq. (3), does not converge. The appearance of non-converging states is also a consequence of the well-known mixing of the discrete states of the donor with the continuum of states belonging to the unbound Hamiltonian in the presence of electric field. Thus, within our model we are facing the problem of separating "reliable" from "unreliable" states, which is done on the base of their convergence, i.e., localization of the states. Away from the discontinuities the ground-state wave function is represented by well converging series, even in the case of large fields (cf. the lower panels in figure 2). Furthermore, we may compare the overall shape of our ground-state dependence with that of Ref. [4]. If we downshift our curve by $\sim 12 \mathrm{meV}$ (account for the chemical shift is absent in our calculation, but present in [4]), the two curves, apart from discontinuities, essentially coincide (not shown) up to $\sim 3.4 \mathrm{MV} / \mathrm{m}$. After this value the ground-state energy of Ref. [4] decreases faster than ours and the disagreement between the two curves reaches $\sim 1.4 \mathrm{meV}$ at $4 \mathrm{MV} / \mathrm{m}$. We are also able to trace the energies (and amplitudes) of the two excited states anti-crossing the ground-state energy at $\sim 1.8 \mathrm{MV} / \mathrm{m}$ and $3.1 \mathrm{MV} / \mathrm{m}$. Despite the poor description of the states in our model, for the two criticalfield values considered $(1.8$ and $3.1 \mathrm{MV} / \mathrm{m})$ the two anti-crossing states exhibit linear energy-versus-field dependence with equal slopes below (for the higher-energy) and above (for the lower-energy state) the anti-crossing region (cf. figure 1), which is qualitatively similar to the result of [4]. Let us assume for the moment that our critical electric fields $(1.8$ and $3.1 \mathrm{MV} / \mathrm{m})$ correspond to the critical-field values of 2.45 and 3.25 MV/m of Ref. [4], respectively. This assumption is reasonable, because in both calculations these are the two highest critical values of the electric field. We may then compare the slopes of the linearlydependent on the field state at each resonance. These slopes are $-13.9 \mathrm{meV} \cdot \mathrm{MV}^{-1} \cdot \mathrm{m}($ near $1.8 \mathrm{MV} / \mathrm{m})$ and $-8.9 \mathrm{meV} \cdot \mathrm{MV}^{-1} \cdot \mathrm{m}$ (near $3.1 \mathrm{MV} / \mathrm{m}$ ) in our calculation, to be compared with $-16.3 \mathrm{meV} \cdot \mathrm{MV}^{-1} \cdot \mathrm{m}$ and $-13.2 \mathrm{meV} \cdot \mathrm{MV}^{-1} \cdot \mathrm{m}$ obtained from figure 1 of Ref. [4] near their critical fields of $2.45 \mathrm{MV} / \mathrm{m}$ and 3.25 $\mathrm{MV} / \mathrm{m}$, respectively. Thus, for the lower critical field our slope is by $15 \%$ lower than that of Ref. [4], but the discrepancy reaches $33 \%$ for the higher one. It is not difficult to see that in all considered cases the energies of Ref. [4] are lower than ours, indicating that the accuracy of our results is inferior and deteriorates with increasing electric field. Therefore, the experimental data will be compared with the results of Ref. [4]. We notice that the smaller slopes of our states considered above tend to move the anticrossing points to higher fields. The fact that both considered critical fields in our calculation lie below the corresponding fields of Ref. [4] is entirely due to the large upshift of our ground-state energy due to neglect of the chemical shift. Thus, the use of our model is restricted solely to the following qualitative consideration regarding the behaviour of the wave functions of the anti-crossing states near one of the critical fields.

As a rough measure of the ionization probability one may use the squared amplitude at the origin (at the donor nucleus), which is easily calculated within our model using the set of coefficients $\left\{\alpha_{n l}^{1 s}\right\}$. The squared amplitudes of the $1 s$ state and the nearest in energy excited state are plotted in figure 1(b), illustrating the concept of "exchange of the character of the states" near the critical fields $\sim 1.8$ and 3.1 $\mathrm{MV} / \mathrm{m}$. In both regions of anti-crossing the amplitude of the ground state decreases and vanishes with increasing field, but at the same time the amplitude of the anti-crossing state increases and approaches the previous value of the $1 s$ state, i.e., the states exchange their characters (the bound state becomes unbound, and vice versa). Similar behaviour of the wave-function amplitudes is observed in Ref. [4] near their critical fields of 2.45 and $3.25 \mathrm{MV} / \mathrm{m}$. 


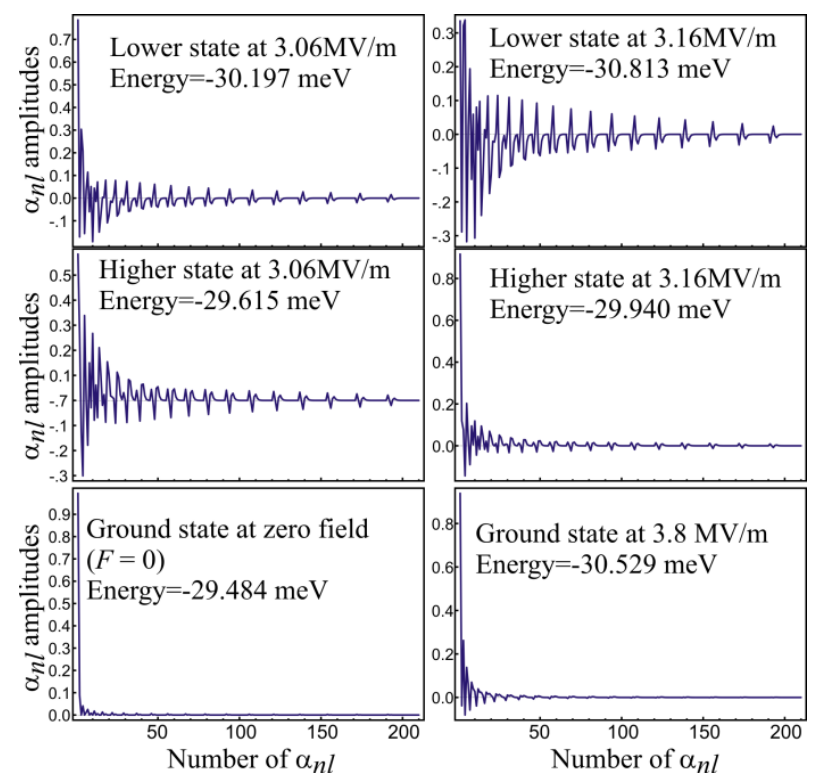

Figure 2. Illustration of the coefficients in the series expansion, Eq.(3), for the ground state and the nearest excited state at two values of the field near the resonance at $3.1 \mathrm{MV} / \mathrm{m}$, as indicated in each panel. The two bottom panels are reference for the appearance of the ground state at zero field (left) and $3.8 \mathrm{MV} / \mathrm{m}$ (right), as discussed in text.

Let us consider the behaviour of the sets $\left\{\alpha_{n l}\right\}$ of the two anti-crossing states, e.g. near the resonance around $3.1 \mathrm{MV} / \mathrm{m}$. The two sets describing the ground state and the anti-crossing excited state are plotted in figure 2 in the following manner. The coefficients $\alpha_{n l}$ are enumerated by assigning number 1 to the state with $(n=1, l=0)$, followed by $(n=2, l=0)$, then $(n=2, l=1),(n=3, l=0)$, etc., and displayed as a line plot for better visibility. The top four panels illustrate the exchange of the characters between the lower state and the higher state. Before the resonance, at $3.06 \mathrm{MV} / \mathrm{m}$, the lower state (top left panel) is still well localized, i.e., the first coefficient $\alpha_{10}$ has dominant amplitude, while the higher state (middle left panel) is significantly less localized. At $3.16 \mathrm{MV} / \mathrm{m}$ the situation is reversed, now the lower state (top right panel) is delocalized, whereas the higher state (middle right) is now better localized. The lower two panels in figure 2 are displayed as a reference for the pattern representing the ground state at zero field (left) and at $3.8 \mathrm{MV} / \mathrm{m}$; it illustrates a good convergence of the series in Eq. (3) in both cases. The convergence is obviously affected near the resonance (cf. the top four panels in figure 2), which is another indication for resonantly increased ionization probability; the expansion series is not sufficiently large to represent either of the two anti-crossing states at the resonance, which is a sign of strong delocalization of the states. However, well-localized ground state exists both below and above the critical field, corroborating the concept of resonant ionization.

We notice that equation (1) is similar to the equation describing hydrogen atom in electric field (with $f(\theta) \equiv 1$ ), which has been treated in the literature in numerous ways (see, for instance, [9] and the references therein). However, in the vast literature devoted to the Stark effect in hydrogen it is generally accepted that the lifetimes of the ground and the excited states decrease (hence, their ionization probabilities increase) monotonically with the electric field. To the best of our knowledge, Ref.[4] is the first account on possibility of sudden ionization at a certain "critical" electric field due to anti-crossing of the states. Despite the shortcomings of our model, the results are qualitatively similar to the more advanced theory of Ref. [4], and both models suggest that more than one critical value of the electric field may exist, at which resonant ionization of the donor electrons may occur (explicitly, near the electric-field values 2.45 and $3.25 \mathrm{MV} / \mathrm{m}$ of Ref. [4]). The term "resonant" is used here to express that the ionization probability actually peaks in a small region around the critical value of the electric field, meaning that it is not necessarily a monotonic function of the field. The experimental results presented in the next section 
are in better agreement with the concept of resonant ionization rather than smooth monotonic increase of the ionization probability with the field, the latter being actually valid away from the critical field values.

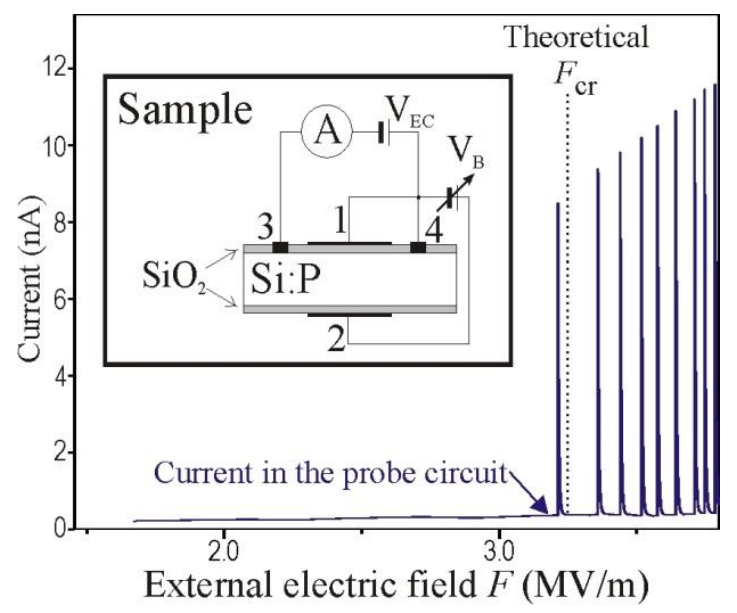

Figure 3. Experimental data representing the dependence of the current in the probe circuit (contacts 3 and 4) on the electric field in the bulk of the sample, created by applying voltage across the large contacts (1 and 2). The insert illustrates the sample and its connections. The theoretical critical field of Ref. [4] is also denoted. Note that the horizontal axis represents the external electric field; the electric field inside the sample never exceeds the critical one, as discussed in text.

\subsection{Experimental results and discussion}

In order to check experimentally the concept of resonant ionization of the donors in electric field we designed a sample of phosphorus-doped $\mathrm{Si}$ (doping concentration $[\mathrm{P}] \approx 10^{14} \mathrm{~cm}^{-3}$ ), arranging four contacts on the sample as shown in the insert of figure 3 . The large circular ( $3 \mathrm{~mm}$ in diameter) contacts 1 and 2 on the top and bottom surfaces serve as plates of a capacitor and are aligned on top of each other. Applying voltage to these contacts results in quite homogeneous electric field in the bulk between the contacts, which is readily calculated knowing the thickness of the sample $(\sim 80 \mu \mathrm{m}$ in our case). Note that the capacitor contacts are isolated from the silicon bulk by a $\sim 3000 \AA$-thick oxide layer, the purpose of which is to prevent the so called space-charge limited (injection) current in this circuit [10]. Another probe circuit is arranged by the auxiliary contacts ( 3 and 4 in figure 3, directly to the Si surface), which is operated at low voltage (standard $1.5 \mathrm{~V}$ battery) and the purpose of which is to test the conductivity of the sample. In order to ensure that all donor electrons are "frozen" in the ground state, the measurements are conducted at low temperature, $2 \mathrm{~K}$, with the sample immersed in pumped liquid helium. The dependence of the current in the probe circuit on the electric field in the sample is presented in figure 3 . We observe a sharp increase in the current when the electric field reaches the value $F_{\text {cr }} \approx 3.2 \mathrm{MV} / \mathrm{m}$, followed by a rather slow decrease. The increase in the current is so sharp that our measuring equipment (HP4140B pA-meter) is too slow to follow it, hence the apparent magnitude of $\sim 10 \mathrm{nA}$ may well be an underestimate of the true current peak.

The sharp increase followed by slow decrease, and the repeated oscillations in the current can be understood from the following consideration. When the electrons are released from the donors they drift to the anode (electrode 1 in figure 3), leaving behind ionized donor atoms. As a consequence, an internal electric field builds up opposing the externally applied field. The result is that the field inside the Si bulk falls below the critical value and the donors are now capable to capture electrons again. Conditions for current flow between contacts 3 and 4 (cf. figure 3) are now present. On one hand, the ionized donors attract electrons from the negative contact 3 and, on the other hand, the electrons accumulated near the anode are capable to drift towards the positive contact 4 . The filling of the donors by electrons supplied from the current in the probe circuit is a slow process, hence the slow current decrease after the peak. As the population of neutral donors increases the internal field opposing the external one decreases and 
eventually the resulting field in the bulk reaches again the critical value. At this point the electrons are released again from the donors and the cycle repeats again. From this consideration it follows that within the present sample design the field inside the Si bulk never exceeds the critical field, no matter what voltage in excess is applied at the electrodes 1 and 2 . The ramp of the external voltage used is $5 \mathrm{mV} / \mathrm{s}$, and the voltage is kept constant for several hours after the maximum value corresponding to about $4.2 \mathrm{MV} / \mathrm{m}$ is reached. The current oscillations are observed during all this time, as long as the field is above the critical value. The increasing frequency of the oscillations with higher external voltages is due to the fact that the critical field can be reached even when the internal opposing electric field is not completely quenched, i.e., not all donors are neutralized, if the external electric field is strong enough to compensate the residual internal one.

We now compare the experimental value $F_{\text {cr }} \approx 3.2 \mathrm{MV} / \mathrm{m}$ with the results of the advanced theoretical calculation of Ref. [4]. In this work the behaviour of the wave function around the anticrossing at $F_{\mathrm{cr}} \approx 2.45 \mathrm{MV} / \mathrm{m}$ is discussed in detail, but figure 1 of Ref. [4] reveals another anti-crossing at about $3.25 \mathrm{MV} / \mathrm{m}$, suggesting existence of another critical field $F_{\text {cr }} \approx 3.25 \mathrm{MV} / \mathrm{m}$. Obviously, there is a good agreement of the experimental result $\left(F_{\text {cr }} \approx 3.2 \mathrm{MV} / \mathrm{m}\right)$ with the theoretical one, $F_{\text {cr }} \approx 3.25 \mathrm{MV} / \mathrm{m}$ of Ref. [4], hence we tentatively ascribe the experimental value to this particular theoretical value. In our opinion, the critical fields of 2.45 and $3.25 \mathrm{MV} / \mathrm{m}$ of Ref. [4] may correspond to the discernible resonances near $F \approx 1.8$ and $3.1 \mathrm{MV} / \mathrm{m}$ in our calculation, as already mentioned in Sec. 3.1. Another confirmation of the suggested correspondence between the two theories is the nearly identical gap of 0.32 $\mathrm{meV}$ for the resonance at $1.8 \mathrm{MV} / \mathrm{m}$ (in our calculation) and the one at $2.45 \mathrm{MV} / \mathrm{m}$ (in the calculation of [4]). Indeed, the value of the gap depends on the strength of interaction between the states involved, which in turn is determined by the overlap of the corresponding wave functions in the spatial region around the donor. Both calculations (our and that of Ref. [4]) contain the low-order terms in the expansion of the corresponding wave function, which are responsible for the above-mentioned overlap, which might explain why the energy separations between the anti-crossing states are in agreement in these two calculations, whereas the critical fields are not. Thus, the results of both calculations are qualitatively similar at higher fields in what concerns the presence of discontinuities in the dependence of the groundstate energy on the field (cf. figure 1 of Ref. [4] with our figure 1). However, as discussed in Sec. 3.1, the many resonances at low fields (in our calculation) should be considered as artefacts.

Finally, it is reasonable to ask the question why the resonance at $2.45 \mathrm{MV} / \mathrm{m}$ predicted in [4] is not observed? This is not well understood at present, but it may be speculated that the reason involves the measurement temperature $(T \approx 2 \mathrm{~K})$ and the energy gaps between the anti-crossing states discussed above. In our calculation the two largest gaps are about $0.32 \mathrm{meV}$ for the resonance $\sim 1.8 \mathrm{MV} / \mathrm{m}$ and $\sim 0.55 \mathrm{meV}$ for the resonance $\sim 3.1 \mathrm{MV} / \mathrm{m}$ (cf. figure 1), and the former was shown to agree well with the gap near $2.45 \mathrm{MV} / \mathrm{m}$ of Ref. [4]. The gap is not specified for the critical field of $3.25 \mathrm{MV} / \mathrm{m}$ in Ref. [4], but it may be assumed that it will be larger than the one at $\sim 2.45 \mathrm{MV} / \mathrm{m}$, possibly close to $0.5 \mathrm{meV}$ as in our calculation. At $T \sim 2 \mathrm{~K}$ the thermal energy $k T \approx 0.17 \mathrm{meV}$ is of the order of the separation between the two anti-crossing levels at $2.45 \mathrm{MV} / \mathrm{m}$. Hence, the higher level will start being thermally populated when its energy approaches that of the ground state. Its population is further facilitated by the presence of intermediate level (cf. the insert of figure 2 in Ref. [4]); the latter is absent in our calculation when only fully-symmetric states are considered. As a result, when the lower state becomes unbound with increasing field, its population may decrease below the limit for detecting the ionized electrons. Other processes like releasing an electron from one donor and recapturing it into the higher (bound) state of another can be envisaged, too. For the resonance near $3.2 \mathrm{MV} / \mathrm{m}$, the gap between the anti-crossing states is probably too large to be overcome by thermal excitation at $2 \mathrm{~K}$, hence the electrons are "forced" to follow the lower branch on the anti-crossing curve, which becomes unbound at larger fields as discussed above. Thermalinduced hoping from the lower (unbound) to the higher (bound) state might be an admissible reason for the non-observation of the resonance at $2.45 \mathrm{MV} / \mathrm{m}$ in the experiment at $2 \mathrm{~K}$. The above scenario implicitly assumes the presence of at least one binding state with very small ionization probability (i.e., the presence of capturing centres) at all fields around the critical one. The analysis conducted in Ref. [4] for the critical field of $2.45 \mathrm{MV} / \mathrm{m}$ seems to confirm this. Indeed, below the critical field the lowest of the 
three considered states (called ground state in [4]) is well localized in the potential well surrounding the impurity, whereas the highest (called second excited state in [4]) is delocalized. The situation is reversed above the critical field, whereas at the critical field both states are well localized, albeit a small but nonnegligible probability to find the electron outside the potential well also exists, as depicted in their figure 2 [4]. It is likely that the ratio between the integrated amplitudes outside and inside the potential well is significantly larger for the critical field at $3.25 \mathrm{MV} / \mathrm{m}$ than that for $2.45 \mathrm{MV} / \mathrm{m}$, stipulating the observation of resonant ionization. However, the above speculation does not provide complete explanation. More comprehensive understanding would require analysis of the ionization probabilities for the involved states in the frame of adequate theory, which would account also for mixing of the donor states with the states of the continuum induced in the presence of electric field. To the best of our knowledge, such theory is not available yet and the reasons for observation of only one ionization threshold at $\sim 3.2 \mathrm{MV} / \mathrm{m}$ need further investigation.

\section{Conclusion}

In conclusion, resonant ionization of the $\mathrm{P}$ donor in silicon at electric field $\sim 3.2 \mathrm{MV} / \mathrm{m}$ is observed experimentally and is in good agreement with the theoretical result of Ref. [4]. The experimental result is discussed in terms of interaction of the states in the frame of a simplified model which, however, is in qualitative agreement with the advanced calculation of Ref. [4]. The theoretical results suggest discontinuities in the ground-state vs. electric field dependence associated with possibility for resonant ionization of the donor electrons at low temperature around two different values of the applied electric field (2.45 and $3.25 \mathrm{MV} / \mathrm{m})$, which is in contrast to the commonly accepted concept of monotonic increase of the ionization probability with the applied electric field. However, only the second critical value is in agreement with our experiment and the absence of another resonance near the critical field $2.45 \mathrm{MV} / \mathrm{m}$

still needs to be understood. Since the effect of resonant ionization seems to be a common property of the Coulomb potential, its analogue should exist also for acceptors, and might provide a new approach to resolving the well-known issue of p-type doping in III-nitrides and $\mathrm{ZnO}$, in addition to the implications to the design of the quantum computer often discussed in the literature.

\section{References}

[1] Kane B E 1999 Nature 393133

[2] Smit G D J, Rogge S, Caro J and Klapwijk T M 2004 Phys. Rev. B 70035206

[3] Friesen M 2005 Phys. Rev. Lett. 94186403

[4] Debernardi A, Baldereschi A and Fanciulli M 2006 Phys. Rev. B 74035202

[5] Landau L D and Lifshitz L M 1991 Quantum Mechanics: Non-Relativistic Theory (Pergamon Press, Oxford)

[6] Ivanov I G, Stelmach A, Kleverman M and Janzén E 2006 Phys. Rev. B 73045205

[7] Faulkner R A 1969 Phys. Rev. 184713

[8] Janzén E, Stedman R, Grossmann G and Grimmeiss H G 1984 Phys. Rev. B 291907

[9] Fernández F M 1996 Phys. Rev. A 541206

[10] Lampert M A and Mark P 1970 Current Injection in Solids (Academic Press, New York) 